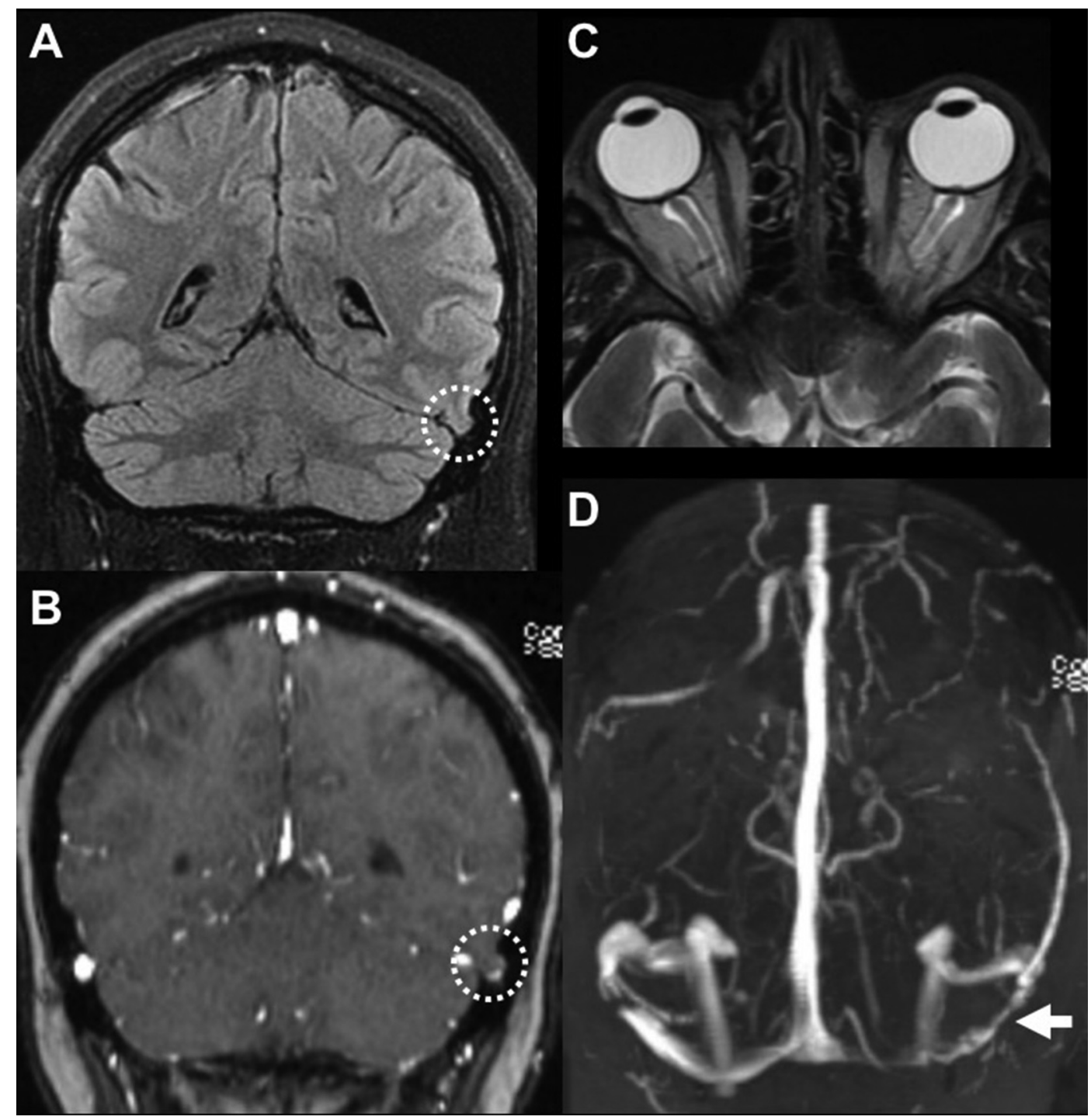

Abstract E-059 Figure 1 Intracranial hypertension in the setting of a left transverse sinus cephalocele. (A,B) Coronal FLAIR and T1 post-contrast MR images show a small herniation of the left occipital lobe into the transverse sinus (dotted circle). (C) Axial T2 MR image shows flattening of the posterior globes and protrusion of the optic nerve heads as well as prominent subarachnoid space in the optic nerve sheathes. (D) MR venogram shows bilateral, left greater than left, transverse sinus stenosis, most pronounced at site of cephalocele (white arrow).

into dural venous sinuses in 16 patients identified on MRI and discuss their imaging findings, possible causes, and relationship to the patient's symptoms. All patients were examined with MRI including pre- and post-contrast T1- and T2weighted sequences. With respect to brain herniations we documented their location, signal intensity, size, presence of arachnoid granulation, and associated dural venous sinus stenosis. We then reviewed clinical records in an attempt to establish if any symptoms were related to the presence of these herniations. 262 patients who presented to our institution's Pulsatile Tinnitus Clinic over a three year period were examined. 16 patients had brain herniations into dural venous sinuses (age range 25-79). 11 patients had unilateral temporal or occipital herniations into the transverse sinus or the transverse-sigmoid sinus junction, respectively. Three patients had unilateral cerebellar herniations into the transverse sinus. One patient had bilateral temporal herniations into the transversesigmoid sinus junction. One patient had bilateral occipital and cerebellar herniations into the transverse sinuses. Arachnoid granulations were seen in association with 13 of the herniations. Focal dural venous sinus stenosis was associated with 15 of the 18 herniations. In 9 of 16 patients (56\%) symptoms matched side of brain herniation. 9 of 16 patients $(56 \%)$ had intracranial hypertension. Brain herniations into dural venous sinuses are uncommon incidental findings with an uncertain relationship to pulsatile tinnitus and intracranial hypertension. Disclosures J. Villanueva-Meyer: None. L. Eisenmenger: None. V. Shah: None. K. Meisel: None. M. Amans: None.

\section{E-060 A CASE OF TWIG-LIKE OR UNFUSED MIDDLE CEREBRAL ARTERY AND REVIEW OF LITERATURE}

${ }^{1}$ A Schnure*, ${ }^{2}$ A Sweidan, 'I Yuki, 'S Suzuki. 'Neurosurgery, UC Irvine, Orange, CA; ${ }^{2}$ Neurology, UC Irvine, Orange, CA

10.1136/neurintsurg-2019-SNIS. 135

Introduction Twig-like or unfused middle cerebral arteries (MCA) are rare vascular anomalies. During embryologic development, it is hypothesized that the primitive MCA is made up of arterial twigs which develop into the definitive MCA. 
Failure of these arterial twigs to fuse result in the twig-like or unfused MCA.

Methods A retrospective single case report of twig-like MCA with literature review. The patient established care at our institution in 6/2018 and still follows with us.

Results A 55-year-old woman with a past medical history of hyperlipidemia and tobacco use presented in the outpatient setting with an electrical-like sensation over her right neck. She denied focal weakness, speech difficulty, visual problems or numbness. There was no history of trauma or neck manipulation. She has a family history of stroke and aneurysm. She was evaluated by magnetic resonance angiography (MRA) of the head which showed a small right internal carotid artery (ICA) and high-grade stenosis of the right ICA and right MCA. Computed tomography (CT) angiography demonstrated small caliber of the entire right ICA and critical stenosis versus occlusion of the right ICA and MCA with distal reconstitution compatible with a moyamoya pattern with lenticulostriate collateralization. CT brain perfusion demonstrated a significant reduction in cerebral blood flow of the entire right MCA territory without a decrease in cerebral blood volume. Cerebral angiography revealed a right twig-like MCA with contralateral ICA cavernous segment aneurysm. The patient has been treated conservatively with aspirin and atorvastatin.

The figure 1 illustrates the right internal common carotid injection with $3 \mathrm{D}$ rotational angiography reconstruction image (A) and AP subtraction image (B). The right M1 segment of the middle cerebral artery has a plexiform arterial network that is consistent with an unfused middle cerebral artery. There are no moyamoya vessels in the basal region and no occlusion in the terminus segment of the internal carotid artery. The twig-like artery can be traceable to the distal M1 or proximal M2 segment. There is no intracranial atherosclerotic change.

Conclusion Twig-like or unfused MCA are rare vascular anomalies that may be mistaken for moyamoya disease. The detailed trans-femoral cerebral angiogram interpretation of the twig-like MCA depicts distinctive differences from the findings of moyamoya disease.

Disclosures A. Schnure: 4; C; General Electric, CYTRX, Portola Pharmaceuticals. A. Sweidan: None. I. Yuki: None. S. Suzuki: None.

\section{E-061 TRANSVERSE VENOUS SINUS STENOSIS AS A TREATABLE CAUSE OF IDIOPATHIC INTRACRANIAL HYPERTENSION: AN UNDER-RECOGNIZED ENTITY}

P Ramakrishnan*, C Berry, S Leinfelder, W Leesch, J Sanderson. Neurovascular Specialists, Riverside Regional Medical Center, Newport News, VA

\subsection{6/neurintsurg-2019-SNIS. 136}

Introduction and purpose Interventional treatment for transverse sinus stenosis with venous sinus stenting is emerging as a viable, and minimally invasive treatment for medically refractory idiopathic intracranial hypertension (IIH). However, this therapeutic intervention remains under-recognized and often accompanied by limited diagnostic evaluation and neurointerventional referral. We present the commonly employed neuroimaging paradigm in the South Eastern Virginia population with IIH, and seek to highlight areas of improvement to better stratify patients based on disease mechanism, incorporating neuroanatomic and pathophysiologic considerations.

Materials and methods We conducted a retrospective chart review within our health system from January 2018 to September 2018, extracting clinical data from 70 cases. From this data, 59 were categorized as confirmed $\mathrm{IIH}$, with 11 presently undergoing workup. The following variables were analyzed in those with confirmed IIH: patient demographics, presenting symptoms, papilledema, and neuroimaging.

Results Fifty-seven female and two male patients with confirmed IIH ( $\mathrm{n}=59)$ with a mean age of 38 and BMI of 39.2 exhibited headache (98\%), vision changes (80\%), pulsesynchronous tinnitus (48\%), and papilledema (64\%) at disease onset. Median cerebrospinal fluid (CSF) opening pressure was $36 \mathrm{~cm} \mathrm{H} 2 \mathrm{O}$. Extent of diagnostic evaluation was assessed and retrospective review of MR venography (MRV) conducted. Twenty-eight (47.4\%) patients in this cohort underwent MRV imaging, amongst whom 20 (71.4\%) exhibited asymmetric venous sinuses or evidence of venous sinus stenosis. Two of the 20 patients (10\%) were referred for neurointerventional evaluation based on suspected transverse sinus stenosis as the mechanism for refractory IIH. Both patients underwent successful transvenous sinus stenting with resolution of transvenous sinus pressure gradient, and clinical symptoms.

Conclusion In this retrospective cohort of IIH, fewer than half of these patients underwent MRV to evaluate for transverse
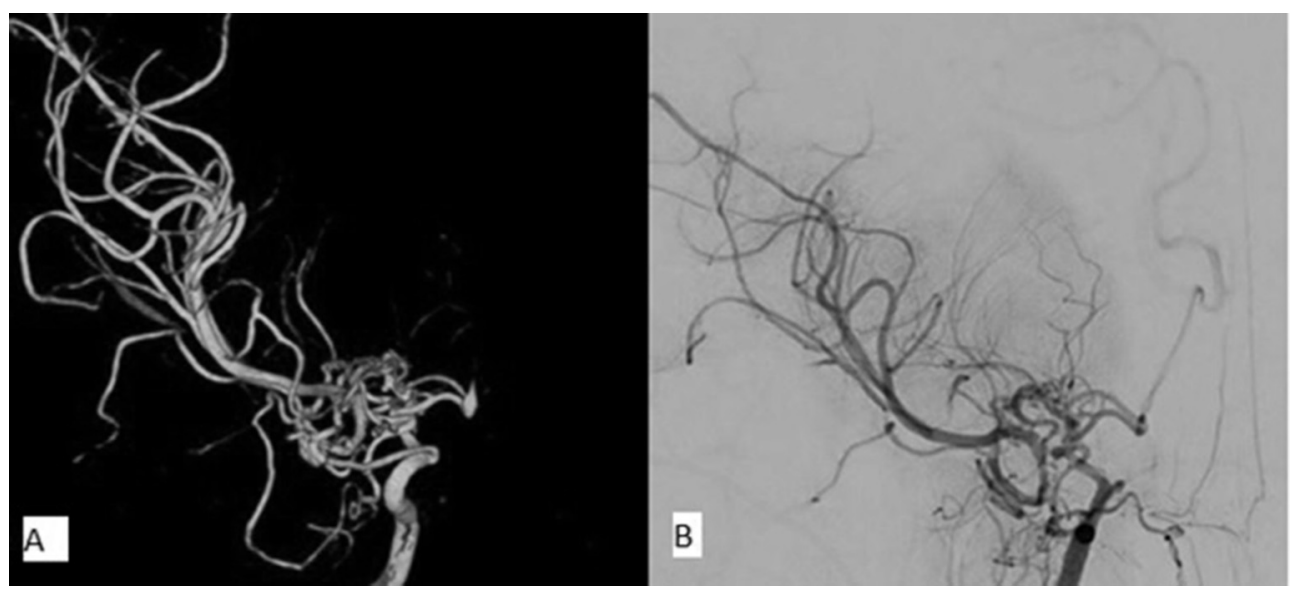

Abstract E-060 Figure 1 